Int. J. Speleol. 10 (1978), pp. $331-350$

\title{
Notes éco-éthologiques et parasitologiques sur les Chiroptères cavernicoles du Shaba (Zaïre)
}

\author{
par
}

\author{
M. ANCIAUX DE FAVEAUX
}

\section{SUMMARY}

Eco-ethological and parasitological data on the cavernicolous (hiroptera in Shaba (Zaire)

After a brief analysis of the elements of the cavernicolous microclimate in Shaba, the author cmphasizes the possibility of relations between ecology and parasitism. He then reviews the 19 species of Chiroptera that live in the subterranean field in Shaba, pointing out their environmental habitat, behaviour and parasites.

\section{INTRODUCTION}

Le espèces cavernicoles sont celles qui fréquentent des biotopes souterrains lithiques: grottes, abris sous-roche, pseudo-grottes formées par l accumulation d'éboulis ou de blocs rocheux, fissures et crevasses rocheuses, terriers d'Oryctéropes; nous y englobons les souterrains artificiels tels que: caniveaux d'évacuation d'eau sous les routes, caves, tunnels, galeries minières abandonnées, etc...

Au Shaba, nous avons effectué des observations biologiques, en savane, dans 45 grottes et dans 7 cavités artificielles, surtout dans les régions de I ikasi, Mwadingusha, Lubudi et Lubumbashi (ANCIAUX, 1965a). La plupart des grottes ont été explorées avec l'aide de la Société Spéléelogique du Katanga, de 1956 à 1960; et, avec quelques amis dévoués, de 1961 a 1965.

Cette étude ne traite ni de la reproduction ni de la léthargie des (hiroptiones qui font l'objet d'autres publications (ANCIAUX, 1976b \& 1978).

Le détail de nos récoltes, (avec lieux et dates) est mentionne ailleurs (ANCIAUX, 1972a \& 1978). Notre matériel a été déposé dans divers Musées:

- BMNH (British Museum Natural History): détermination. R. W. HAYMAN

- IRSNB (Institut Royal des Sciences Naturelles de Belgique): déterminations X. MISONNE \& W. VERHEYEN

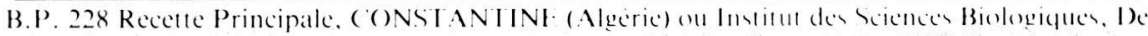

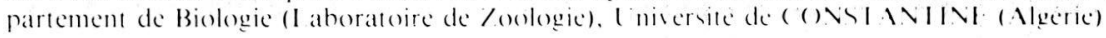


- MHNG (Muséum d'Histoire Naturelle de Genève): détermination V. AELLEN

- MHNP (Muséum National d'Histoire Naturelle, Paris): détermination J. DORST

- MRAC (Musée Royal de l'Afrique Centrale, Tervuren): détermination R. W. HAYMAN

- SMF (Senckenberg Museum Francfort a.M.): détermination H. FELTEN.

\section{ANAIYSE DES ELEMENTS DU MICROCLIMAT CAVERNICOLE}

I es facteurs écologiques sont importants à préciser, car ils conditionnent la présence ou l'absence des Chiroptères et de leurs parasites, leur répartition géographique, leur habitat, leur rythme nycthéméral, leur biologie reproductrice.

\section{Obscurité}

Chez les espèces internes, surtout troglophiles, l'obscurité est relativement importante: sauf, notamment, pour Rousettus aegyptiacus leachi qui accepte de giter dans une certaine pénombre. Ce facteur n'est toutefois pas primordial (contrairement à l'avis de VERSCHUREN, 1957: 44), car plusieurs espèces cavernicoles se réfugient parfois dans des abris moins obscurs, tels que des hangars et des maisons (par exemple, Hipposideros ruber et Miniopterus schreihersi natalensis).

\section{Humidité}

Dans les grottes, un haut degré hygrométrique (souvent voisin de la saturati(n) est nécessaire, d'une part, pour lutter contre la dessication des membranes alaires et, d'autre part, pour favoriser l'état léthargique.

Dams les grottes du Shaba, presque partout et durant toute l'année, il y a 95 à 10(0) d'humidité, sauf dans quelques grottes sèches (Baya, Kasowena et Kiwahishi).

\section{Température (en degrés centigrades)}

I n regle genérale, la température d'une grotte correspond à la température excírieure moyenne annuelle (celle-ci est de $20,5^{\circ}$ à $21,4^{\circ}$ à I ubumbashi).

Nor mesures thermiques dans les parties profondes des grottes ont été éffectuée plus a l’ones de l ubumbashi et dams des régions d’altitude supérieure.

a) Cirottes de grandes dimensions 


\begin{tabular}{lllll}
\hline Grottes & Dates & T.air & T.eau & T.extér. \\
\hline Kalomoni (Kakontwe) & $15 / 5 / 1957$ & $23^{\circ}$ & $23,5^{\circ}$ & \\
& $12 / 7 / 1957(9 \mathrm{~h})$. & $24^{\circ}$ & & $9,5^{\circ}$ \\
& $11 / 9 / 1957(10 \mathrm{~h})$. & $22^{\circ}$ & $23,4^{\circ}$ & \\
& $8 / 5 / 1965$ & $21,5^{\circ}$ & $22^{\circ}$ & \\
Grotte au Ruisseau (Tantara) & $4 / 6 / 1960$ & $22,5^{\circ}$ & \\
Gouffre Albert (Tantara) & $18 / 2 / 1958$ & $24^{\circ}$ & \\
Grotte Kondo (Kakanda) & $31 / 5 / 1962$ & $22^{\circ}$ & \\
Grotte Kiwakishi & $20 / 7 / 1958$ & $23^{\circ}$ & $24^{\circ}$ \\
Grottes de Lubudi: & & & \\
Kyantapo & $29 / 7 / 1957$ & $24^{\circ}$ & \\
Kyamakonde & $18 / 7 / 1962$ & $20^{\circ}$ & \\
Mulonga & $26 / 7 / 1962$ & $19,5^{\circ}$ & \\
Lusolo & $16 / 7 / 1962$ & $20^{\circ}$ & \\
Kyasala & $28 / 7 / 1957$ & $22^{\circ}$ & \\
Kyasala & $21 / 7 / 1962$ & $20^{\circ}$ & \\
Kabayaboya & $24 / 4 / 1962$ & $22^{\circ}$ & \\
Kaboyaboya & $23 / 7 / 1962$ & $20,5^{\circ}$ & \\
\hline
\end{tabular}

\section{Remarques}

Les températures de l'air varient entre 19,5 et $24^{\circ}$ au cours de l'année, et se répartissent comme suit: février $\left(24^{\circ}\right)$, avril $\left(22^{\circ}\right)$, mai $\left(21,21,5,22\right.$ et $\left.23^{\circ}\right)$, juin $\left(21,5^{\circ}\right)$, juillet $\left(19,5,20,22,23\right.$ et $\left.24^{\circ}\right)$ et septembre $\left(22^{\circ}\right)$.

L'isolement microclimatique de ces grottes n'est pas totalement réalisé, car on note des températures plus basses en saison sèche (surtout de mai à juillet) où les nuits sont très fraîches à l'extérieur (jusqu'à 3 et $2^{\circ}$ ).

\section{Grottes moins profondes}

\begin{tabular}{lllll}
\hline Grottes & Dates & T.air & T.eau & T.ext. \\
\hline Mwanga (Pompéré) & $30 / 6 / 1964$ & 13 à $15^{\circ}$ & & \\
Katembavikulu (Pempéré) & $31 / 8 / 1958$ & $23^{\circ}$ & $19^{\circ}$ & \\
Katembavikulu (Pempéré) & $30 / 6 / 1964$ & $16^{\circ}$ & $18,5^{\circ}$ & \\
Etienne (Mulungwishi) & $27 / 12 / 1959$ & $23,5^{\circ}$ & & \\
Etienne (Mulungwishi) & $10 / 1 / 1960$ & $23,2^{\circ}$ & & $23,2^{\circ}$ \\
\hline
\end{tabular}




\section{Remarques}

Dans le massif de Pempéré, les températures notées dans la grotte Mwanga étaient de $13^{\circ}$ en bas de la cheminée d'accès, et de $15^{\circ}$ dans la salle terminale; dans la grotte Katembavikulu, il y a un fossé d'eau (nappe phréatique), avec $\mathrm{pH}$ entre 7,5 et 7,2, respectivement en eau claire et en eau boueuse, le 3 octobre 1959: c'est une nouvelle station d'une forte population d'Amphipodes troglobies Ingolfiella leleupi Ruffo, 1951.

La grotte Etienne constitue un biotope favorable à Histoplasma capsulatum Darling, 1906 (ANCIAUX, 1960a \& b, 1964a; BOVY, $1960 \&$ 1961; PATTYN \& DELVILLE, 1960): elle a une humidité de 99 à 100\%, alors que dans les trois autres grottes de Mulungwishi, le taux d'humidité est de 85 à $90 \%$, ce qui pourrait expliquer l'absence d'Histoplasmes.

Il convient de noter que les grottes Mwanga et Katembavikulu (Pempére) abritent des Histoplasmes, car des Noirs, travaillant à extraire du guano, ont été contaminés; il en est de même pour les grottes Kyantapo, Kyasala et Lusolo (L.ubudi) où, à la suite d'une exploration en août 1957, nous avons contracté une histoplasmose pulmonaire bénigne (ANCIAUX, 1964a).

\section{Conclusion}

I 'obscurité, la température et l'humidité ont une importance discutable. Parmi les facteurs géographiques, la forêt dense humide est plus favorable aux Mégachiroptères, tandis que la savane est davantage colonisée par les Microchiropteres.

I es facteurs écologiques, dont l'incidence directe sur le choix de l'abri diurne "t sur le comportement des Chiroptères est incontestable, sont: absence de courants d'air, proximité de l'eau, faible luminosité et caractères propres du gîte (suivant les espèces).

ICOIOCiIE FI PARASITISME (ANCIAUX, 1972b)

\section{Influence du biotope sur les parasites}

a) Vulle sur: les microorganismes (Salmonelles et Virus), les Protozoaires sanguins (Nucteria, Trupanosoma), les Hémiptères Polyctenidae, les Acariens endoparavites (Demodicidac, Ereynetidae, (iastronyssidae, Teinocoptidae).

b) Discurathle sur: les Protoroaires sanguins (Hepatocystis, Plasmodium, Polychromophilus), les Helminthes, les Acariens Trombidiformes (Myobiidae. 
Psorergatidae) et Sarcoptiformes (Chirodiscidae et Rosensteiniidae).

c) Plus ou moins déterminante sur: les Insectes (Hémiptères Cimicidae, Siphonaptères, Diptères Nématocères et Diptères Pupipares), les Acariens Ixodoidea, Mesostigmata, Trombidiformes (Leeuwenhoekiidae et Trombiculidae) et Sarcoptiformes (Sarcoptidae).

\section{Nombre de parasites en fonction de l'écologie des hôtes}

Chez les Chiroptères de savane, troglophiles et grégaires, le parasitisme est intense, se manifestant par une infestation double, triple ou davantage: un même parasite chez deux (trois ou plus) genres d'une même famille de Chiroptères.

La répartition des ectoparasites s'explique davantage par la biologie des ( Chiroptères que par leurs affinités systématiques. Elle dépend surtout de facteurs écologiques: nature des gîtes occupés par les Chiroptères, macrobiotope (forêt ou savane), vie sociale (solitaires, petits groupes, colonies monospécifiques ou plurispécifiques). Les grottes, les fissures de rochers, les arbres creux sont souvent riches en matières organiques (guano) qui favorisent le développement des larves: ces microbiotopes facilitent le passage des parasites entre diverses espèces de Chiroptères.

L e domaine forestier semble généralement moins favorable aux grandes colonies de Microchiroptères qui ny trouvent pas les conditions optimales de nourriture; il abrite beaucoup d'espèces solitaires ou formant des groupes restreints. Cela peut expliquer un parasitisme plus réduit qu'en savane.

\section{A. FAMILLE PTEROPODIDAE}

1. Rousettus (Rousettus) aegyptiacus leachi (A. Smith, 1829)

Matériel Anciaux: avril, juin et octobre 1957, avril, août et novembre 1958. mars et octobre 1959, 11 of et 39 Q, dans la grotte Mwanga (Pempéré). (collections MHNG \& MRAC). Dans une colonie de plusicurs milliers d'individus, présente à peu près toute l'année (absence noté les 21/4/1958 et 21/6/1959): les Roussettes sont isolées ou forment de petits essaims dans lat /one médianc d'une grande diaclase où une ouverture supérieure répand une Jumiere tamisèe. Sex-ratio: $30 \%$ of et $70 \%$ ơ. Espèce peu sociable.

Dans la grotte Kalomoni (Kakontwe), 6 cadavres sur le lac souterrain el quelques individus solitaires sur les parois, le 17 juillet 1956.

Dans la grotte de Kandu (I ubudi), au début de juillet 1962, une colonie avait subi un enfumage (procédé utilisé par les indigenes pour capturer les Rousset- 
tes à des fins gastronomiques...).

\section{Parasites}

Plasmodium (V.) rousetti Van Riel \& Hiernaux, 1951: cas douteux, grotte Kalomoni (ANCIAUX, 1958 \& 1965b).

Afrocimex leleupi Schouteden, 1951: grotte Kalomoni (SCHOUTEDEN, 1951: L.ELEUP, 1956): grotte Mwanga: nous avons aussi observé cette Punaise, en grand nombre, sur les parois et dans des fissures; nous avons noté des formes crithidiennes chez cet Hémiptère qui est probablement vecteur de Trypanosomes (ANCIAUX, 1965b).

Thaumapsylla b. breviceps Rothschild, 1907: grotte Mwanga (COOREMAN, 1950); SM1T, 1954; LEIEUP, 1956; ANCIAUX, 1965b).

Eucampsipoda africana Theodor, 1955: grottes Kalomoni et Mwanga (THFODOR, 1955; I 1:LEUP, 1956; ANCIAUX, 1965b).

Brachytarsina africana (Walker, 1849): grottes Kalomoni et Mwanga (JOBI.IN(i, 1954: I I:LEUP, 1956; ANCIAUX, 1965b).

Brachytarsina a. alluaudi (Falcoz, 1923): grotte Kalomoni (JOBI IN( i, 1954) et grotle Nlwangal (dét. THEODOR).

Ancystropus zeleborii Kolenati, 1856: grotte Mwanga (BENOIT, 1958b.).

Ancystropus leleupi Benoit, 1959: grottes Kalomoni et Mwanga (BFNOIT, 1959; AN( 1 (I). 1965b.).

Meristaspis lateralis (Kolenati, 1856): grottes Kalomoni et Mwanga (BFN()IT, 1958b; IN (IAUX, 1965b).

Acariens indéterminés (Coll. Anciaux: MRAC): grotte Mwanga.

Acariens (indéterminés) dans le guano (Coll. Anciaux: MRAC ): grolle Mwangal.

2. Lissonycteris angolensis angolensis (Bocage, 1898)

Matériel Anciaux (MRAC): deux femelles capturées, le 1/12/1957, dans une colonie d'une vingtaine d'individus, dans la grotte de Kalumbu (Kabolela), loin de Miniopterus schreibersi natalensis.

Colonie absente, le 1/7/1959.

\section{Parasites}

Penicillidia (C.) fulvida Bigot, 1885 (dét. THEODOR).

Dipseliopoda biannulata (Oldroyd, 1953) (dét. THEOD()R).

Acariens indéterminés (Coll. Anciaux: MRAC).

\section{B. FAMILLE EMBALLONURIDAE}

3. Taphozous (Taphozous) perforatus sudani (Thomas, 1915)

Matériel Anciaux (IRSNB, MHNG, MRAC, SMF): en juin 1957, juin ct août 1958, août 1962, 9 d́ et 17 o, dans la grotte Mwanga (Pempéré), au sein d'unc colonie de plusieurs centaines d'individus occupant une petite chambre fort obscure (niveau supérieur); cette colonie est absente durant la saison des pluice (d'octobre à mars). Son gîte est très proche de l'aire d'un couple de Tryo alha: cette chouette ne semble pas s'attaquer à cette espece.

Espèce farouchement indépendante. Sex-ratio: $350^{\circ}$ \& et 65\% o o.

Dans la grotte Kasowena (Pempéré), seulement der mâles, le 28/6/1964.

Parasites (dams la grotte Mwanga)

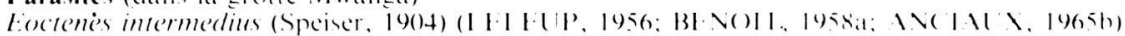

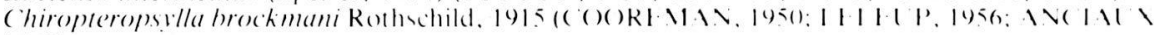
196.5b). 
Brachytarsina a. alluaudi (Falcoz, 1923) (JOBLING, 1954).

Steatonyssus n.sp. (dét. BENOIT)

Alabidocarpus taphozous Fain, 1971 (FAIN, 1971).

Acariens indéterminés (Coll. Anciaux: MHNG).

\section{C. - FAMILLE NYCTERIDAE}

4. Nycteris thebaica E. Geoffroy, 1818

Matériel Anciaux: une ọ (MHNG), 29/9/1957, associée à Miniopterus schreibersi natalensis et à Rhinolophus swinnyi, dans la grotte au Ruisseau (Tantara). Un of (BMNH), 22/4/1959, solitaire, dans la grotte de Kondo (Kakanda). Un of (SMF), 16/7/1962, associé à Rhinolophus simulator et à Rh. swinnyi, dans la grotte Lusolo (Lubudi). Dans des caniveaux sous la route de Lubumbashi vers Kasenga: au km 60, 1 of (MRAC), 3/7/1956; au $\mathrm{km} \mathrm{67,} 1$ of (MRAC), 8/6/1956. Un ơ (MRAC), 12/7/1956, dans terrier d'oryctérope, Mont Mukwene (Lubumbashi). Dans un tunnel sous l'atelier de forge de la Mission Catholique de Kapolowe, 1 of, 1 q et 2 n.nés of (MRAC), 1 of et 1 q (SMF), 18/10/1956, ainsi qu'une o (SMF), 8/9/1962, dans une colonie d'une vingtaine d'individus très éveillés, suspendue à un plafond de bois, en pleine obscurité et dans une atmosphère très sèche et très chaude. Sex-ratio: $47 \%$ of et $53 \%$ o.

\section{Parasites}

Eoctenes nycteridis (Horvath, 1910): caniveau sous la route de Lubumbashi vers Kasenga (AN(IAUX, 1965b) et grotte au Ruisseau (dét. BENOIT). Diptères pupipares indéterminés (Coll. Anciaux: MHN(i): grotte Lusolo.

\section{Nycteris macrotis macrotis Dobson, 1876}

Synonyme: Nycteris aethiopica luteola Thomas, 1901 (KOOPMAN, 1965; KOCK, 1969; ADAM \& HUBERT, 1976).

Matériel Anciaux: une o (MRAC), 26/3/1956, dans un drain de ciment sous la route de Lubumbashi vers Kasenga, au km 36 (Bulaya). Un of (MRAC), $12 / 3 / 1956$, dans la grotte de Baya, isolé au milieu d'une colonie de Rhinolophus hildebrandti. Un of et 2 o (IRSNB), 1 of et 1 q (SMF), 2/8/1960, au sein d'une colonie d'une cinquantaine d'individus, dans une galerie minière à Shinkolobwe. Dans des caniveaux d'évacuation d'eau sous la route de Lubumbashi vers Sakania, près de la ferme Droogmans (Keyberg), 8 ot $6 . q$ (BMNH, MHNG, MHNP, MRAC, SMF) d'octobre à décembre 1955 ainsi qu'en mars et en juin 1956.

Habituellement, en petites colonies de 5, 10 à 20 individus. Cohabitation fréquente avec des Hystricidae (porcs-épics). Sex-ratio: $47 \%$ of et 53\% 0 .

\section{Parasites}

Nicleria medustormis (iarnham \& Heisch, 1953: Keyberg (I.IPS \& RODHAIN, 1956; AN('1AIX. 1958: KRAMPIT/ \& AN(1AUX: 1960).

Irypanosema thomasi l ip \& Rodhain. 1956: Keyberg. 
Vampyrolepis sp. (oeufs dans fèces): Keyberg.

Eoctenes nycteridis (Horvath, 1910): Keyberg (BENOIT, 1958a; ANCIAUX, 1965b) et Shinkolobwe (Coll. Anciaux: IRSNB).

Microtrombicula nycteris Jadin \& al., 1955: Keyberg (ANCIAUX, 1958).

Psorergatoides nycteris Fain, 1959: Keyberg (Fain, 1959a).

Acariens indéterminés (Coll. Anciaux: IRSNB): Shinkolobwe.

\section{D. - FAMILLE RHINOLOPHIDAE}

6. Rhinolophus landeri lobatus Peters, 1852

Matériel Anciaux: 9 of et 7 o, en juillet 1956 et 1957 (MRAC), grotte Kalomoni. 12 of et 5 o (BMNH, IRSNB, MHNP, MRAC), en juin 1956, en octobre et en décembre 1959, dans les grottes de Mulungwishi. Un of et 1 g. (MRAC), 1/7/1959, dans la grotte Kalumbu (Kabolela). Une o (MRAC), 1/5/1958, grotte de la Mura (Kambove). Un ó (IRSNB) et une o (SMF), 21/9/1960, grotte de Kondo. 2 q (SMF), 18/12/1957, dans une galerie le long d'une conduite forcée, à Mwadingusha.

Colonies de 20 à 50 individus, associées à Hipposideros ruber et à divers Rhinolophidae. Sex-ratio: $60 \%$ of et $40 \%$ o.

\section{Parasites}

Penicillidia (C.) pachymela Speiser, 1901: Mulungwishi (VERMEIL, 1965).

Brachytarsina africana (Walker, 1849): grottes Kalomoni (ANCIAUX, 1965b) et Mura (dét. THE(ODOR).

Raymondia (R.) simplex Jobling, 1955: Mulungwishi (VERMEIL, 1965; THEODOR, 1968b).

Raimondia (R.) boquieni Vermeil, 1965: Mulungwishi.

Acariens indéterminés (Coll. Anciaux: IRSNB): grotte de Mulungwishi.

\section{Rhinolophus hildebrandti Peters, 1878}

Matériel Anciaux: en juillet 1956, en janvier, février et juillet 1957, 9 d et 4 o (MHNG, MHNP, MRAC), grotte Kalomoni. Six f́f, en juin 1957, 1958 et 1959, en août 1958 et 1960 (MHNG, MRAC, SMF) et une o (MRAC), 11/6/1958, grotte Mwanga. Une oq (MRAC), 11/11/1957, 6 o (IRSNB) et 8 o (SMF), 4/6/1960, grotte au Ruisseau (Tantara). Un ơ (MRAC), 1/7/1959, grotte Kalumbu. Un of (MRAC), 1/5/1958, gouffre Swanepoel (Kambove). Un of et 9 o (MRAC), 22/4/1959, 1 of et 7 q (IRSNB), 1 of et 1 q (SMF), grotte de Kondo, 21/9/1960. Une $q$ (SMF), 11/11/1959, 7 of et 9 q (IRSNB), 6/12/1959, grotte Tshamakele (Mwela-Pande). Dans les grottes de Lubudi: un of (MRAC), 25/7/1957, grotte de Kandu; douze of (IRSNB, MHNG, SMF), en juillet 1957 et 1962, et 8 (BMNH, MHNG, MRAC), en avril 1956, en juillet 1957 et 1962, grotte Kyasala; un of (MHNG), 23/7/1962, grotte Kaboyaboya. En mars et en mai 1956, 13 \& et 29 q (MHNG, MHNP, MRAC), grotte de Baya. Une of (IRSNB), 22/9/1960, galerie minière de Kakanda.

Isolés ou en colonies ( 10 à 30 individus assez écartés les uns des autres). Aussi dans la grotte de Kiwakishi (Coll. LELEUP, juillet 1958). Sex-ratio: $40 \%$ of et $6\left(0^{0}\right)$ q $\%$. 
Les colonies sont souvent éloignées des autres Chiroptères (Hipposideridae, Minioptères, autres Rhinolophidae). Dans la grotte Mwanga, Rhinolophus hildebrandti manifeste un comportement agressif envers $R h$. clivosus zuluensis et $R h$. swinnyi. Si l'exiguïté de la grotte de Baya les mettait à proximité des Minioptères, ces deux espèces - par suite de nos visites en mars 1956 - ont formé des colonies nettement distinctes dans les galeries basses d'un puits de sondage, à plus d'un $\mathrm{km}$ de la grotte de Baya.

Il semble bien que ces groupements plurispécifiques répondent davantage à des exigences écologiques semblables plutôt qu'à une attraction sociale d'une espèce pour une autre (BROSSET, 1974).

Baguage: deux ớ et 6 oq, 7 ơ et 9 o provenant de la grotte de Baya, ont été relâchés respectivement à Lubumbashi, les 12 mars et 23 juillet 1956; un mâle a été repris à Baya, à la fin mai 1965 (distance $35 \mathrm{~km}$ sud), ce qui témoigne d'une fidélité au gîte habituel (ANCIAUX, 1960c).

\footnotetext{
Parasites

Nycteria congolensis (Krampitz \& Anciaux, 1960): grotte Kalomoni (KRAMPITZ \& ANCIAUX. 1960; GARNHAM, 1973).

Afrocimex leleupi Schouteden, 1951: grottes Kyasala et Tshamakele (FAIN, 1972).

Penicillidia (C.) fulvida Bigot, 1885: grotte Tshamakele (VERMEIL, 1965).

Penicillidia (C.) pachymela Speiser, 1901: grotte Kyasala (dét. THEODOR).

Nycteriphila schmidli scotti (Falcoz, 1923): grotte de Baya (ANCIAUX, 1965b).

Phthiridium hoogstraali (Theodor, 1957): grottes du Shaba (THEODOR, 1957): Baya (AN CIAUX, 1965b), Tantara et Tshamakele (VERMEIL, 1965).

Phthiridium scissum rhodesiense (Theodor, 1957): grottes de Tantara et Tshamakcle (VI:RMIIII. 1965).

Brachytarsina africana (Walker, 1849): grottes de Baya, Kalomoni et Kyasala (ANCIAUX. 1965b, THEODOR, 1968b) et Tshamakele (VERMEIL, 1965).

Raymondia (R.) waterstoni Jobling, 1931: grotte Kyasala (THEODOR, 1968b).

Raymondia (R.) setiloba Jobling, 1954: grottes Baya, Kyasala (THEODOR, 1968b), Pempere. Tantara et Tshamakele (VERMEIL, 1965).

Ascodipteron brevior Maa, 1965: grotte de Baya (THEODOR, 1968b).

Diptères Pupipares indéterminés (Coll. Anciaux): grottes Kyasala, Pempere, Tantara (MRAC ( ) et Kaboyaboya (MHNG).

Diptères Mycetophilidae indéterminés (Coll. Anciaux: MHN(i): en phorésie, dams grolle kyasala (dét. STUCKENBERG, Muséum Vienne).

Spinturnix semilunaris De Meillon \& Lavoipierre, 1944: grotte Kyasala (AN( IAUX. 1965 ).

Alabidocarpus $m$. megalonyx (Trouessart, 1895): grottes de l.ubudi (I:AIN, 1971).

Labidocarpoides congoensis Fain. 1970: grottes de Lubudi (FAIN, 197()a \& b. 1971).

Acariens indéterminés (Coll. Anciaux): grottes de Kondo, Pempere, Ishamakcle (IRSNB), Kalumbu, Lubudi et Pempéré (MRAC).
}

\section{Rhinolophus blasii empusa K. Andersen, 1904}

Matériel Anciaux: un mâle (SMF) solitaire au milieu d'une colonie de Hipposideros ruber, grotte de Kondo, 31/5/1962.

\section{Parasites}

Nicteridocoptes elndhoveni Fain, 1959: Moba, Shaba, 23 + 1954. (NRAC) (1 AIN. 19596).

9. Rhinolophus clivosus zuluensis K. Andersen, 19()4 
Matériel Anciaux: deux o, 3/4/1957 et 1 o, 21/6/1959 (MRAC), grotte Mwanga; une o (MRAC), 1/5/1958, gouffre Swanepoel; une 우 (IRSNB), 21/9/1960, grotte de Kondo.

Seulement des femelles isolées ou à proximité d'autres Rhinolophidae.

Parasites (petite grotte de Lubudi, coll. N. Leleup, novembre 1948)

Psorergatoides rhinolophi Fain, 1959 (FAIN, 1959a).

Nycteridocoptes eyndhoveni Fain, 1959 (FAIN, 1959b).

10. Rhinolophus simulator K. Andersen, 1904 (Coll. SMF)

Le matériel Anciaux provenant des même grottes est attribué par V. AELLEN à Rhinolophus swinnyi, mais H. FELTEN confirme sa première diagnose («in littera», 23 avril 1971): un of et 1 우, 11/6/1960, grotte Kalomoni; un of et 1 우, 7/8/1960, grotte Mwanga; un ớ, 30/6/1962, grotte au Ruisseau; grottes de Lubudi: un of et 2 , 18/7/1962, Kyamankonde; deux d et 3 , $26 / 7 / 1962$, Mulonga; un of et $5+q, 16 / 7 / 1962$, Lusolo; quatre of et $1 q 23 / 7 / 1962$, Kaboyaboya. Grotte Kasoma: quatre of et 3 , $13 / 8 / 1962$.

Petites colonies de 10 à 20 individus, cohabitant avec Rhinolophus hildebrandti, Rh. swinnyi, des Hipposideridae et des Minioptères, dans les grottes Kalomoni, Pempéré, Tantara, Lubudi et Kasoma.

Sex-ratio: égalité des sexes.

\section{Parasites}

Brachytarsina africana (Walker, 1849): grotte Mwanga (VERMEIL, 1965).

Raymondia (R.) waterstoni Jobling, 1931: grottes Mwanga (VERMEIL, 1965) et Kasoma (THIODOR, 1968b).

Diptères pupipares indéterminés (Coll. Anciaux: MHNG): grotte Kyamakonde.

\section{Rhinolophus fumigatus exsul (Anderson, 1905)}

Matérial Anciaux: quatre ó et 7 q (MRAC), 22/4/1959, grotte de Kondo, dans une colonie d'une cinquantaine d'individus, en compagnie de Hipposideros ruber et de Rhinolophus hildebrandti; un et 4 Q (MRAC) et 2 q (SMF), 18/12/1957, galerie le long d'une conduite forcée à Mwadingusha, dans une colonie de 20 à 30 individus, près de Rhinolophus landeri lobatus. Sex-ratio: $30 \%$ of et $70 \%$ o.

Parasites (grottes de I ubudi)

Psorergatoides rhinolophi Fain, 1959 (FAIN, 1959a).

victeridocoptes elndhoveni Fain, 1959 (FAIN, 1959b).

\section{Rhinolophus swinnyi Gough, 1908}

Matériel Anciaux: un of et 3 of, 8/7/1957, et 1 \&, $12 / 7 / 1957$ (MHNG), 1 \&, $11 / 6 / 1960$ ), et 1 o , 20/5/1962 (IRSNB), grotte Kalomoni; une \& (MHNG), 19/6/1957, et 2 \& (MRAC), 21/6/1959, grotte Mwanga; un of (BMNH), 27/12/1959, grotte de Mulungwishi; deux $q$ (MHNG) et 1 (MRAC), 
29/9/1957, grotte au Ruisseau (Tantara); dans grottes de Lubudi: un of (MHNG), 29/7/1957, Kyantapo; une q (MHNG), 18/7/1962, Kyamankonde; un of (MHNG), 25/7/1957, Kandu; deux of et 1 \& (MHNG), 30/7/1957, deux of et 1 (MHNG), 30/7/1957, 2 of (MRAC), 1/5/1959, 1 of et 1 o (IRSNB), 26/7/1962, Mulonga; une $q$ (BMNH), 3 of et 3 o (MHNG), 9 of et 10 q (MRAC), 28/7/1957, 2 ơ et 7 o (IRSNB), 2 ơ et 4 q (MHNG), 16/7/1962, Lusolo; une o (MHNG) et 1 ( $(\mathrm{MRAC}), 15 / 4 / 1956,1$ of et 1 o (MHNG) et 6 of (MRAC), 28/7/1957, Kyasala; un of et 2 o (IRSNB), 3 of et 1 o (MHNG), 23/7/1962, Kaboyaboya; un of 3 o (MHNG), 13/8/1962, grotte Kasoma. Petites colonies de quelques dizaines d'individus, associées à des Hipposideridae, Minioptères, Nycteridae et Rhinolophidae.

Sex-ratio: $42 \%$ of et $58 \%$ o.

\section{Parasites}

Nycteria congolensis (Krampitz \& Anciaux, 1960): grotte Kyasala (KRAMPITZ \& ANCIAUX, 1960).

Penicillidia (C.) pachymela Speiser, 1901: grotte Kyasala (THEODOR, 1968a).

Phthiridium hoogstraali (Theodor, 1957): grotte Mulonga (dét. THEODOR).

Phthiridium ovale (Theodor, 1957): grottes Kaboyaboya et Kasoma (THEODOR, 1968a).

Brachytarsina africana (Walker, 1849): grottes Kaboyaboya, Lusolo et Kasoma (THEOD)(OR. 1968b).

Raymondia (R.) alulata Speiser, 1908: grotte Lusolo (THEODOR, 1968b).

Raymondia (R.) waterstoni Jobling, 1931: grottes Kaboyaboya, Kyasala, I usolo, Kasoma (THEODOR, 1968b), et Mulonga (dét. THEODOR).

Eyndhovenia euryalis (Canestrini, 1884): grotte Mulonga (BENOIT, 1957).

Spinturnix walkerae Zumpt \& Till, 1954: grotte Mulonga (BENOIT, 1958c; AN(IAUX, 1965b). Spinturnix sp.: grotte Kyasala (Coll. Anciaux: MHNG).

Steatonyssus benoiti Till \& Evans, 1964: grotte Mwanga (TILL \& EVANS, 1964).

Acariens indéterminés (Coll. Anciaux: MHNG): grottes de Lubudi et de Tantara.

\section{E. - FAMILLE HIPPOSIDERIDAE}

13. Hipposideros commersoni gigas (Wagner, 1845)

Dans la grotte Kalomoni, deux individus suspendus sous une voûte très basse, à moins d'un mètre de la surface d'un plan d'eau, le 5/7/1948 (L.EI.EUP, 1956).

14. Hipposideros ruber ruber (Noack, 1893)

Synonyme: Hipposideros caffer centralis Andersen, 1906.

Matériel Anciaux: en juin-juillet 1956, février et juillet 1957, et juin 1960), 16 \& et 8 o (MHNG, MHNP, MRAC), grotte Kalomoni; une of (MRAC) 28/6/1964, grotte Mwanga; une of (MHNG), 1 of et 1 of (MRAC), 28/6/1956, grotte de Mulungwishi; quatre of et 1 q (MRAC), 15/6/1958, 2 \& et 2 o (SMF), 4/6/1960, grotte au Ruisseau; douze of (MRAC), 22/4\%1959, 10 of et 3 \% (IRSNB), 7 of et 1 \& (MHNG), 2 of et 6 of (SMF), 31/5/1962, grotte de Kondo; dans grottes de Lubudi: six of et 4 o (MRAC), 2/5/1959, Mpopola; en mars- 
avril 1956, en juillet 1957, en avril et juillet 1962, 13 d et 14 o (IRSNB, MHNG, SMF), Kyasala; un of et 1 (SMF), 24/4/1962, Kaboyaboya. 3 of et 4 o (IRSNB), 2 of et 3 q (MHNG), 3 of et 2 o (SMF), 13/8/1962, grotte Kasoma; un o (MHNG), 25/8/1957, grotte Tshamalenge; un of (MRAC), 3/6/1958, galerie minière de Likasi.

Individus rarement isolés. Petites colonies (5 à 10 animaux) ou moyennes (de 20 à 50 chauves-souris). Groupements plurispécifiques: avec Hipposideridae, Minioptères et Rhinolophidae. Sex-ratio: $55 \%$ of et $45 \%$ o.

Grotte de Kiwakishi: cinq o et 3 o, 7/7/1948, 1 ó et 1 o, octobre 1948 (Coll. S. Frechkop: IRSNB).

Baguage: aucune reprise sur un total de 26 spécimens bagués à Kakontwe, Lubudi et Mulungwishi (ANCIAUX, 1960c).

\section{Parasites}

Nycteria congolensis (Krampitz \& Anciaux, 1960): grottes Kalomoni er Kyasala (KRAMPITZ \& ANCIAUX, 1960).

Trypanosoma (M.) leleupi Rodhain, 1951: grotte Kalomoni. Vecteur possible: Afrocimex leleupi Schouteden, 1951 (ANCIAUX, 1965b).

Prosthodendrium pseudocystosphincter Dubois, 1964: Albertville.

Penicillidia (C.) fulvida Bigot, 1885: grotte Kalomoni (ANCIAUX, 1965b).

Penicillidia (C.) pachymela (Speiser, 1901): grottes Kalomoni, Kiwakishi, Kyasala, Mulungwishi (THEODOR, 1957) et Tantara (VERMEIL, 1965).

victeriphila schmidli scotti (Falcoz, 1923): grotte Kalomoni (LELEUP, 1956; ANCIAUX, 1965b).

Phthiridium scissum sudanicum (Theodor, 1957): Mitwaba (THEODOR, 1957).

Brachytarsina africana (Walker, 1849): grottes de Lubudi et de Pempéré (JOBLING, 1954; LEI.LUP, 1956; ANCIAUX, 1965b) et grotte Kalomoni (VERMEIL, 1965).

Ravmondia (R.) waterstoni Jobling, 1931: grottes de Lubudi, Pempéré (JOBLING, 1954; LEI FUP, 1956; ANCIAUX, 1965b) et Kalomoni (THEODOR, 1968b).

Raimondia (R.) seminuda Jobling, 1954: grottes Kalomoni, Lubudi (JOBLING, 1954; LELEUP, 1956), Kyantapo (ANCIAUX, 1965b), Kondo, Kaboyaboya, Kyasala et Kasoma (THEODOR, 1968b).

Ratmondia (R.) setiloba Jobling, 1954: grottes Kalomoni, Lubudi (JOBLING, 1954; LELEUP, 1956) et Kyamakonde (ANCIAUX, 1965b).

Rarmondia (R.) simplex Jobling, 1955: grotte de Mulungwishi (THEODOR, 1968b).

Ascodipteron brachythecum Theodor, 1968: grottes Kyasala et Mulungwishi (THEODOR, 1968b). I a "myiase" signalée par nous (ANCIAUX, 1958 \& 1965b) est, en fait, un Ascodipteron colkyte (AFIIFN, 1970).

Dipteres pupipares indeterminés (Coll. Anciaux): grottes Kalomoni, Kasoma, Kyasala, Kondo (.MHN(i), I ikasi, I usolo, Mpopola, et Tantara (MRAC).

Paraperig/ischrus moucheti (Till, 1958): grotte Kalomoni (BENOIT, 1958c; ANCIAUX, 1965b). Porergatoides hipposideros Fain, 1959: grotte Kalomoni (FAIN, 1959c).

Aariems indetermines (Coll. Anciaux): grotte Kondo (IRSNB), Kaboyaboya, Kasoma, Kasala, Kondo (MHNCi) c' Tantara (MRAC).

15. Cloeotis percivali australis Roberts, 1917 (HAYMAN, 1960 : 168)

Matériel Anciaux: un $\mathfrak{f}$ et 1 o (MRAC) et 1 \& (BMNH), 15/6/1958, 1 of et 1 o (SMF), 4/6/1960, grotte au Ruisseau (Tantara); un of (MHNG), 1 of et 1 q (SMF), 23/7/1962, grotte Kaboyaboya (Lubudi); un o (SMF), 13/8/1962, grotte Kasoma (Parc National Upemba).

Isolés ou en petites colonies. Sex-ratio: $55 \%$ ó et $45 \%$ o. Associé à Hipposide- 
ros ruber, Myotis tricolor et Rhinolophus hildebrandti, grotte au Ruisseau; à divers Rhinolophidae et Minioptères, grotte Kaboyaboya; à Hipposideros ruber, des Rhinolophidae et 2 espèces de Minioptères, grotte Kasoma.

Grotte de Kiwakishi: un of et 1 o, 4/10/1948 (Coll. S. Frechkop: IRSNB).

Parasites

Eoctenes intermedius (Speiser, 1904): grotte au Ruisseau.

Diptère pupipare indéterminé (Coll. Anciaux: MHNG): grotte Kaboyaboya.

Hipposiderobia (H.) cloeotis Fain, 1974: grotte au Ruisseau (FAIN, 1974).

Acarien indéterminé (Coll. Anciaux: MHNG): grotte Kaboyaboya.

\section{F. - FAMILLE VESPERTILIONIDAE}

16. Myotis (Myotis) tricolor (Temminck, 1832)

Matériel Anciaux: un ơ (MHNG), 22/9/1957, grotte Kalomoni; un ớ (SMF), 4/6/1960, grotte au Ruisseau (Tantara); deux $q$ (MHNG \& SMF), 18/7/1962, grotte Kyamakonde (Lubudi); un of (MRAC), 30/7/1957, grotte Mulonga (Lubudi).

Individus isolés dans des crevasses de la voûte ou en compagnie de Minioptères (parfois dans une même fissure): commensalisme étroit avec Miniopterus schreibersi natalensis (un Minioptère of derrière 2 Myotis tricolor $q$, grotte Kyamakonde; un $M$. tricolor avec plusieurs Minioptères, grotte Mulonga). Sex-ratio: $60 \%$ ơ et $40 \%$ o.

Parasites

Pteracarus minutus tricolor Fain, 1974: grotte Mulonga (FAIN, 1974).

\section{G. - FAMILLE MINIOPTERIDAE}

17. Miniopterus schreibersi natalensis (A. Smith, 1834)

Matériel Anciaux: en juin, juillet et octobre 1956, en février, mars, avril et juillet 1957, en janvier et février 1958, en juin 1960, en mai 1962, en janvier 1964 et en mai 1965, 37 d et 35 o, grotte Kalomoni (BMNH, IRSNB, MHN(i, MHNP, MRAC, SMF); en juin 1959 et 1964, 52 of 57 of grotte Mwanga (BMNH, MRAC); trois o (MRAC), 28/6/1964, grotte Kasowena (Pempéré); deux of et 2 (MHNG), $2^{+}$ơ et 3 o (MRAC), 29/9/1957, grotte au Ruisseau; un of (MRAC), 15/6/1958, 1 of et 1 o (SMF), 30/6/1962, grotte aux Serpents (Tantara); quatre of et 13 \& (MRAC), 2 of et 1 \& (SMF), grotte Kalumbu; six of (MRAC), 1 of et 1 \& (SMF) 1/5/1958, gouffre Swanepoel; un of (MRAC), 22/4/1959, grotte Kondo; un of (IRSNB), 6/12/1959, grotte Tshamakele. Dans grottes de Lubudi: un of (MHNG), 3 of et 1 o (MRAC), 29/7/1957, Kyantapo; sept $\mathcal{f}$ (IRSNB) et 3 of (SMF), 18/7/1962, Kyamakonde; trois of et 2 q (MHNG), 16 of et 11 Q (MRAC), 25/7/1957, et 1 of (SMF), 18/7/1962, Kandu; un of et 1 o (MRAC), 2/5/1959, Mpopola; un of (MHN(i) et 2 \& (MRAC), 
30/7/1957, 2 of et 1 q (MRAC), 1/5/1959, Mulonga; une $q$ (MHNG), 2 of et 3 o (MRAC), 28/7/1957, Lusolo; une $q$ (MRAC), 31/3/1956, 1 of et 1 우 (MRAC), 15/4/1956, 2 of (BMNH, MNHG), 1 of et 1 o (MRAC), 28/7/1957, Kyasala; un o (MHNG) et 1 (SMF), 24/4/1962, et 2 of (IRSNB, MHNG), 23/7/1962, Kaboyaboya. En mars et en mai 1956, 26 ó et 18 \& (BMNH, MHNG, MRAC), grotte de Baya; un $\delta$ et 2 o (IRSNB), 1 of (MNHG), 1 of et 3 o (SMF), 13/8/1962, grotte Kasoma; une o (SMF), 18/12/1957, le long d'une conduite forcée à Mwadingusha; en juin et en août 1958, et en avril 1961, 32 ó et $78 q$ (IRSNB, MRAC, SMF), galerie minière de Likasi.

Grosses colonies, souvent en léthargie (ANCIAUX, 1976b), surtout présentes durant la saison sèche (de mai à août): de 500 à 1.000 individus dams la grotte Kalomoni (niveau supérieur); quelques centaines à Pempéré (grotte Mwanga) et dans la galerie minière de Likasi. Durant les autres mois de l'année, individus isolés ou formant de petites colonies.

245 ó et 267 q en collection; les autres, bagués et relâchés, sont au nombre de 341 ơ et 260 q: seulement 4 reprises manifestant une fidélité au gîte diurne ou à la colonie d'origine, avec des distances parcourues de 7 et de $15 \mathrm{~km}$ (cf. déplacements saisonniers: ANCIAUX, 1977). Sex-ratio: $53 \%$ of et $47 \%$ \&.

\section{Parasites}

Pollchromophilus melanipherus (Dionisi, 1899): grottes Kalomoni et Kyasala, maison à Kasapa (près de L ubumbashi) (KRAMPITZ \& ANGIAUX, 1960). Vecteurs possibles: Anopheles rodhaini I eleup \& Lips, 1950: grotte Kalomoni (LELEUP \& LIPS, 1950 \& 1951; ANCIAUX, 1958); Penicillidia (C.) fulvida Bigot, 1885: grotte Kalomoni; Nycteriphila schmidli scotti (Falcoz, 1923): grotte Kalomoni, et Kasapa.

Trypanosoma (S.) pipistrelli Chatton \& Courrier, 1912 (type cruzi): grottes Kalomoni (RODHAIN, 1951; ANCIAUX, 1958) et Kyasala.

litomosa sp. (oeufs embryonnés et microfilaires dans le sang du poumon): grotte Kyasala. 1frocimex leleupi Schouteden, 1951: grottes Kalomoni et Mwanga (ANCIAUX 1965b).

Stricticimex anciauxi Fain, 1972: grotte Kyasala (FAIN, 1972).

Penicilliclia (C.) fulvida Bigot, 1885: grottes Baya, Kakontwe (ANCIAUX, 1965b). Tshamakele (VFRMLII. 1965), Mwanga, Kalumbu, Swanepoel, Kandu et Mpopola (dét. THEODOR).

Victeriphila schmidli scotti (Falcoz, 1923): grottes Baya, Kalumbu, Swanepoel, Kyasala, (Kasapa) (ANC IAUX, 1965b) el grotte Kalomoni (VERMEIL, 1965).

Ratmondia (R.) waterstoni Jobling, 1931: grotte Kaboyaboya (THEODOR, 1968b).

Rarmondia (R.) semimuda Jobling, 1954: grotte Kalomoni (ANCIAUX, 1965b).

Rarmondia (R.) setiloha Jobling, 1954: grotte Kyasala (THEODOR, 1968b).

Ascodipteron theodori Maa, 1965: grotte de Baya (THEODOR, 1968b).

Dipteres Pupipares indéterminés (Coll. Anciaux): galerie minière de Likasi (IRSNB), grottes Kalomoni, Kyamakonde et Tantara (MHNG), grottes Kalomoni, Kyasala et Mwanga (MRAC).

(helonchida (en phoresic): grotte de Kiwakishi (LELEUP, 1958).

Splmarmix semilunaris De Mcillon \& lavoipierre, 1944: grottes Baya et Kalomoni (BENOIT, $1958 \mathrm{~b}$ \& $\because$ AN('IAUX, 1965b)

Maromissus gramulosus (Kolenati, 1856): grottes Baya et Kalomoni (ANCIAUX, 1965b).

lrombicula sp. : grote Kalomoni (ANCIAUX, 1965b).

()ahidecarmus mieticola Fain, 1970): grotte Kalomoni (FAIN, 1971).

1/ahidecarpus mimiopteri lain, 1971: grotes Baya, Kandu et Mulonga (FAIN, 1971)

Acariens indetermines (Coll. Anciaus): grotes Kalomoni, Kandu, Kyamakonde (MHNG), Kalomoni, Kalumbu, Swanepole, Mulonga et Mwanga (MRAC).

18. Miniopterus inflatus rufus Sanborn, 1936 
Matériel Anciaux: deux of et 1 o (MHNG), 13/8/1962, grotte Kasoma (Parc National Upemba), dans des fissures pariétales, en compagnie de Miniopterus schreibersi natalensis.

Dans la grotte de Kiwakishi, 1 f , 5/7/1948, et 1 o, 20/9/1948 (Coll. S. Frechkop: MRAC).

Parasites:

Diptères Pupipares indéterminés (Coll. Anciaux: MHNG).

\section{H. - FAMILLE MOLOSSIDAE}

19. Tadarida (Tadarida) ansorgei (Thomas, 1913)

Matériel Anciaux: deux of et $2 q(\mathrm{BMNH}), 2$ of et $2 q$ (MHNG), 4 of et 8 q (MRAC), 16/7/1957; deux ó (MRAC), 14 det 11 q (bagués), 20/5/1959; capture de 25 individus (non conservés), 17/9/1958. Tous, dans une grosse colonie (plus de 500 individus) occupant les joints de dilatation des murs de l'usine hydroélectrique de Mwadingusha, seulement en saison sèche (de mai à la fin septembre). Animaux fort éveillés durant le jour.

La colonie quitte Mwadingusha au début d'octobre pour gagner un refuge inconnu (arbres creux?) où elle reste jusqu'à la fin avril; ce déplacement est lié à la reproduction.

25 individus bagués ont été relâchés, le 20 mai 1959, à Likasi (distance de 80 $\mathrm{km}$ ): aucune reprise, sauf une femelle (25 mai 1959) dans une maison de Likasi. Sex-ratio: $51 \%$ of et $49 \%$ o.

Parasites (Mwadingusha)

Lagaropsylla anciauxi Smit, 1957: cette Puce a été aussi signalée au (ihana, ches Tudarida (ch.) major (SMIT, 1965).

Chelanyssus spiniferus Till \& Evans, 1966.

Alabidocarpus molossicola Fain, 1970 (FAIN, 1970b \& 1971).

Parasites dans les gîtes de Chiroptères

Blastocrithidia sp.: dans Afrocimex leleupi, grotte Kalomoni (ANCIAUX, 1965b).

Afrocimex leleupi Schouteden, 1951: dans les fissures et sur les parois des grottes Kalomoni, Mwanga, Swanepoel, Kalumbu et Mulungwishi (ANCIAUX, 1964b \& 1965b; FAIN, 1972).

Afrocimex constrictus major Fain, 1972: grottes Kalomoni ct Kalumbu: (FAIN, 1972).

Stricticimex anciauxi Fain, 1972: dans des fissures du gouffre Swanepoel el de la grotle K valsalit. (FAIN, 1972).

Hémiptères indéterminés (Coll. Anciaux: MRAC): grottes de Tantara.

Anopheles (M.) rodhaini Leleup \& Lips, 1950: adultes dans la grotte Kalomoni, et larves dans k «lac» de cette grotte (LELEUP \& LIPS, 1950 \& 1951; I.EIEUP, 1956; I IIIIUP \& ZACiHI, 1960; ANCIAUX, 1964b).

Diptères Psychodidae Phlébotomes indéterminés (Coll. Anciaux): grotles Kalomoni et Swantepoel, Kaboyaboya, Kyamakonde et Kyantapo (MHNG \& MRAC).

Allochernes inexpectatus Heurtault, 1968: grotte Kyasala.

Acariens guanobies indéterminés (Coll. Anciaux: MHN(i \& MRA(') : grottes Kalomoni et Mulungwishi, Kondo, Kaboyaboya, Kyamakonde et Kyantapo. 


\section{Systématique des parasites cités}

\section{A. - PROTOZOAIRES}

\section{Zooflagellates Trypanosomidae}

- Trypanosoma Gruby, 1843 (sous-genres Megatrypanum \& Schizotrypanum)

- Blastocrithidia Léger, 1902

2. Sporozoaires Hémosporidies

a) Plasmodiidae

- Plasmodium Marchiafava \& Celli, 1885 (sous-genre Vinckeia Garnham, 1964)

b) Haemoproteidae

- Polvchromophilus Garnham, 1953

- Nvcteria Garnham \& Heisch, 1953

\section{B. - HEIMINTHES}

1. Trématodes Digenea: Lecithodendriidae Odhner, 1911

- Prosthodendrium Dollfus, 1931

2. Cestodes Hymenolepididae Railliet \& Henry, 1909

- Vampyrolepis Spassky, 1954

3. Nématodes Dipetalonematidae Wehr, 1935

- Lilomosa Van Beneden, 1873

\section{( . - HEXAPODES (INSECTES)}

1. HEMIPTERES HETEROPTERES

a) Cimicidae Latreille, 1804

Cacodminae Kirkaldy, 1899

- Stricticimex Ferris \& Usinger, 1957

Afrociminae Usinger, 1966

- Afrocimex Schouteden, 1951

b) Polyctenidae Westwood, 1874

Polycteninae Westwood, 1874

- Eicclenes Kirkaldy, 1906

\section{SIPHONAPTERES}

Icchnopsyllidae Wahlgren, 1907

Ischnopsyllinae Wahlgren, 1907

- Chiropteropsylla Oudemans, 1908

- I agaropsylla Jordan \& Rothschild, 1921

Ihatumapsyllinae Rothschild, 1907

Thaumapsylla Rothschild, 1907

\section{DIPTERES}

Vematocères 1 atreille, 1825

a) Culicidac l atreille, 1825

Anophelinae Theobald, 1905

Anopheles (sous-genre Myzombia)

b) Mycetophilidac Macquart. 1838

(-) Pechodidac Bigot, 1857

Phleberominate Rondani, 1840

Brachyceres Matcquart, 1834 (IPROBOS(II)H:A = Dipteres pupipares)

a) Victeribidate 11 entwood, 1840

Vucteribiinate 11 esemesed, 184()

Phthiridium Hermann, 18().4 Strlidia Wesmood. 1840) 
- Penicillidia Kolenati, 1863 (sous-genre Cratopenicillidia Speiser, 1908)

- Nycteriphila Grulich \& Povolny, 1955

Cyclopodiinae Theodor, 1967

- Eucampsipoda Kolenati, 1857

- Dipseliopoda Theodor, 1955

b) Streblidae Kolenati, 1863

Ascodipterinae Monticelli, 1898

- Ascodipteron Adensamer, 1896

Nycteriboscinae Speiser, 1900

- Brachytarsina Macquart, 1851

- Raymondia Frauenfeld, 1856

\section{D. - ARACHNIDES}

1. PSEUDOSCORPIONS Chelonethidae

- Cheliferina

- Allochernes

2. ACARIENS (ANCIAUX, $1971 \&$ 1976a)

MESOSTIGMATES Canestrini, 1891

a) Spinturnicidae Oudemans, 1902

Spinturnicinae Oudemans, 1902

- Spinturnix von Heyden, 1826

- Ancystropus Kolenati, 1856

- Meristaspis Kolenati, 1857

- Eyndhovenia Rudnick, 1960

Periglischrinae Benoit, 1957

- Paraperiglischrus Rudnick, 1960

b) Macronyssidae Oudemans, 1936

- Macronyssus Kolenati, 1858

- Steatonyssus Kolenati, 1858

- Chelanyssus Zumpt \& Till, 1953

TROMBIDIFORMES Reuter, 1909 ( = PROSTIGMATES)

a) Myobiidae Megnin, 1877

- Pteracarus Jameson \& Chow, 1952

- Hipposiderobia Dusbabek, 1968

b) Psorergatidae Dubinin, 1883

- Psorergatoides Fain, 1959

c) Trombiculidae Ewing, 1929

- Trombicula Berlese, 1905

- Microtrombicula Ewing, 1950

SARCOPTIFORMES Reuter, 1909 ( = ASTIGMATES)

a) Sarcoptidae Murray, 1877

Notoedrinae Fain, 1968

- Nycteridocoptes Oudemans, 1898

b) Chirodiscidae Trouessart, 1892

Labidocarpinae Gunther, 1942

- Alabidocarpus Ewing, 1929

- Olabidocarpus I awrence, 1948

- Labidocarpoides Fain, 1970 


\section{RÉSUMÉ}

Après une brève analyse des éléments du microclimat cavernicole au Shaba, l'auteur souligne les relations possibles entre l'écologie et le parasitisme. Puis, il passe en revue les 19 espèces de Chiroptères qui peuplent le domaine souterrain du Shaba, en signalant leur répartition, leur comportement et leurs parasítes.

\section{BIBL, IOGRAPHIE}

ADAM F. \& HUBERT B., 1976 - Les Nycteridae du' Sénégal: distribution, biométrie et dimorphisme sexuel. Mammalia, $40: 597-613$.

AELLEN V., 1970 - Communication personnelle. (in littera, 8, décembre).

ANCIAUX de FAVEAUX M., 1958 - Speologica africana. Chiroptères des grottes du Haut-Katanga, Congo Belge. Bull. I.F.A.N., $20 \mathrm{~A}: 263-275$.

- 1960a - Notes biospéléologiques et parasitologiques sur des grottes du Katanga. Bull. Soc. Spéléol. Katanga, 2 : $19-22$.

- 1960b - Contribution à l'étude de l'histoplasmose au Katanga. II. - Considérations écologiques sur l'histoplasmose. Ann. Soc. Belge Méd. Trop., 40 : 298-303.

- 1960c - Baguage des Chiroptères au Katanga. Bull. Soc. Spéléol. Katanga, 2 : 30-33.

- 1964a - L'histoplasmose dans les grottes du Haut-Katanga. Actes III Congr. Int. Spéléol., Vienne (1961), 3 : $7-10,2 \mathrm{pls}$.

- 1964b - La faune des grottes du Katanga. Ibidem, $3: 11-21,2$ cartes.

- 1965a - Les grottes du Katanga. Bull. C.E.P.S.I., Elisabethville, 69 : 77-85, 2 cartes.

- 1965b - Les parasites des Chiroptères. Rôle épidémiologique chez les animaux et l'homme au Katanga. Ann. Parasit. hum. comp., $40: 21-38$.

- 1971 - Catalogue des Acariens parasites et commensaux des Chiroptères. Inst. Roy. Sc. Nat. Belgique. Documents de travail $n^{\circ} 7: X V+451 p$.

- 1972a - Répartition biogéographique et cycles annuels de Chiroptères d'A frique Centrale. Thèse Doctorat Sc: Zool. Univ. Paris VI, 2 vols, 464 p., cartes, tableaux.

- 1972b - Parasitologie des Chiroptères du Continent Africain. Thèse annexe Doctorat Sc. Tool. Unis. Paris VI, 276 p., cartes, tableaux.

- 1976a - Catalogue des Acariens parasites et commensaux des Chiroptères. Sixième et septiène parties. Inst. Roy. Sc. Nat. Belgique, Documents de travail n $7: 452-616$.

- 1976b - La léthargie chez les Chiroptères cavernicoles d'Afrique Centrale. Intern. J. Speleol, $8: 291-303$

- 1977 - Déplacements saisonniers chez Miniopterus schreibersi natalensis (A. Smith, 1833) au Shaba, S.1 Z.Za-

re. Rev. Zool. afr., $91: 721-729,2$ cartes.

- 1978 - Les cycles annuels de reproduction chez les Chiroptères cavernicoles du Shaba (S.F.Zaïre) et du Rwanda. Mammalia, 42: 453-490.

BENOIT P.L.G., 1957 - Essai de classification des Spinturnicidae, Acariens parasites des chauses-souris. Re'l. Zool. Bot. Afr., $56: 382-387$.

- 1958a - Les Polyctenidae du Congo belge. Ibidem, 57 : 68-72.

- 1958b - Contribution à l'étude des Spinturnicidés du Congo belge. Ibidem. $57: 96-1(0)$.

- 1958c - Ibidem, $58: 309-312$.

- 1959 - Un nouveau Spinturnicidé sur chauve-souris frugivore du Congo belge. Ihidem. 59 : 106-10)8.

BOVY P., 1960 - Etude clinique: histoplasmose pulmonaire à forme miliaire. Ann. Soc. Bège Mét. Trop)., 4) 293-297, 2 pls.

- 1961 - Histoplasmose pulmonaire aiguë. Classification des formes de l'histoplasmose. I. he/ge Rudiol.. H4 505-517.

BROSSET A., 1974 - Structure sociale des populations de chauves-souris. J. Psychol. normale et pathol., 71(1): 85-102.

COOREMAN J., 1950 - Suctoria du Congo belge. Rev. Zool. Bot. Afr., $43:$ : 329-335.

DUBOIS G., 1964 - Un nouveau Prosthodendrium de l'Afrique centrale. Res: Zool. Bot. 4/r., 69: $384-388$. FAIN A., 1959a - Les Acariens psoriques parasites des chauves-souris. III. - I e gente Psorergates I yrrell. Bull. \& Ann. Soc. rov. Entom. Belg., $95: 1-4,54-69$.

- 1959b - Ibidem. VI. - Le genre Prosopodectes Canestrini 1897 est composite el doit tomber en synonymie de Notoedres Raillet 1893. Description de Nycteridocoptes evndhoveni n. sp. Acurologia. 1 : 324-334.

- 1959c- Ibidem. IX. - Nouvelles observations sur le genre Psorergates Tyrrell. Bull. \& Ann. Soc. ros: I:ntom. Belg., $95: 232-248$.

- 1970a - Diagnoses de nouveaux I obalgides et I istrophorides. Rer. /.ool. Bot. . 1/r., 81: 271-3(x)

- 1970b - Notes sur quelques nouseaux laxa de la famille (hirodiscidac. Ihichem, 82:28()-284.

- 1971 - I es I istrophorides en Afrique au sud du Sahara. II. - Iamille I ivtrophoridac et ( hirodiscidac. Acta Cool. Pathol. Antverp. 54:1-231.

- 1972 - Notes sur les Punaises parasites de ( hiropteres de la Republique du /anre. ance dencription de deus especes et d'une sous-espece nouvelles. Rev. /ool. Bot. A/r., 85:187.201.

- 1974. Nouselles especen d'Acariens Myobidate d'Afrique au ud du Sahara. Ithedem, 8x: 677-682. 
FELTEN H., 1971 - Communication personnelle. (in littera, 23 avril).

GARNHAM P.C.C., 1973 - The zoogeography of Polychromophilus and description of a new species of a Gregarine (Lankesteria gallieri). Ann. Paras. hum. comp., 48: 231-242.

HAYMAN R.W., 1960 - A note on the bat Cloeotis percivali Thomas. Rev. Zool. Bot. Afr., 61 : 167-172.

HAYMAN R.W., MISONNE, X. \& VERHEYEN W., 1966 - The bats of the Congo and of Rwanda and Burundi. Ann. Mus. Roy. Afr. Centr., Tervuren, in- ${ }^{\circ}$, Sc. Zool., $154: 1-105,20$ pls. : cartes.

JOBLING B., 1954 - Streblidae from the Belgian Congo, with a description of a new genus and three new species. Rev. Zool. Bot. Afr., $50: 89-115$. KOCK D., 1969 - Die Fledermaus-Fauna des Sudan. Abhandl. Senckenberg, Frankfurt a.M., 521 : 1-238, 48 ta-
bles.

KOOPMAN K.F., 1965 - Status of forms described or recorded by J. A. ALLEN in «The American Museum Congo expedition collection of bats". Amer. Mus. Novit., $2219: 1-34$.

KRAMPITZ H.E. \& ANCIAUX de FAVEAUX M., 1960 - Ueber einige Haemosporidien aus Fledermäusen der Höhlen des Berglandes von Katanga. Z.f.Tropenmedizin \& parasit., $11: 391-400$.

LELEUP N., 1956 - La faune cavernicole du Congo belge. Ann. Mus. Roy. Congo belge, Sc. Zool., 46 : 17-78. - 1958 - Récoltes de pseudoscorpions guanophiles phorétiques sur Chiroptères au Congo belge. Folia Scientif.
Afr. centr., $4: 32$.

- \& LIPS M., 1950 - Anopheles rodhaini n.sp. au Katanga. Rev. Zool. Bot. Afr., 43 : 303-308.

- 1951 - Anopheles rodhaini Leleup \& Lips au Katanga. Ibidem, 44 : 169-172.

- \& ZAGHI A., 1960 - A propos d'Anopheles (Myzomyia) faini Leleup et d'Anophelese faini subsp. vanthieli Laarman. Ann. Soc. belge Méd. Trop., $40: 925-931$.

LIPS M. \& RODHAIN J., 1956 - Quelques Hématozoaires de petits Mammifères du Haut-Katanga. Ann. Parasit. hum. comp., 31: 481-488.

PATTYN S.R. \& DELVILLE J.P., 1960 - Contribution à l'étude de l'histoplasmose au Katanga : étude mycologique. Mise en évidence d'histoplasmes dans une grotte. Ann. Soc. belge Méd. Trop., 40 : 303-305. RODHAIN J., 1951 - Trypanosoma leleupi n.sp., parasite de Hipposideros caffer au Katanga. Ann. parasit,
hum. comp., $26: 133-137$.

SCHOUTEDEN H., 1951 - Un genre nouveau de Cimicide du Katanga, Afrocimex leleupi. Rev. Zool. Bot. Afr., 44 : 278-280.

SMIT F.G.A.M., 1954 - New bat-fleas. Parasitology, 44 : 144-156. -1957 - Two new bat-fleas of the genus Lagaropsylla from the Belgian Congo. Rev. Zool. Bot. Afr., $56: 263$ -
268 .

- 1965 - A new bat-flea from Ghana. Ibidem, $72: 135-137$.

THEODOR O., 1955 - On the genus Eucampsipoda Kolenati and Dipseliopoda (Nycteribiidae). Parasitology, 45 : 195-229.

- 1957 - The Nycteribiidae of the Ethiopian region and Madagascar., Ibidem, 47 : 457-543. 1968a - New species and new records of Nycteribiidae from the Ethiopian, Oriental and Pacific regions. Ibi-
dem, $58: 247-276$.

$-1968 \mathrm{~b}-\mathrm{A}$ revision of the Streblidae of the Ethiopian region. Trans. Roy. Entom. Soc. London, 120: 313-373. TILL W.M. \& EVANS G.O., 1964 - A revision of the genus Steatonyssus Kolenati, 1858. Bull. British Museum (N.H.), (Zoology), 11, $8: 513-582$.

- 1966 - The genus Chelanyssus Zumpt \& Till, Acarologia., 8 : 401-414.

VERMEIL C., 1965 - Contribution à l'étude des Nycteribiidae et des Streblidae du Congo-Léopoldville. Raymondia boquieni n.sp. Ann. Parasit. hum. comp., $40: 693-708$.

VERSCHUREN J., 1957 - Exploration du Parc National de la Garamba. Mission H. DE SAEGER. Ecologie, biologie et systématique des Cheiroptères. Inst. Parcs Nationaux Congo belge, Bruxelles, fasc. $7: 1-473$. 